\title{
System dynamics applications for defence combat modelling: preliminary insights from a literature exploration
}

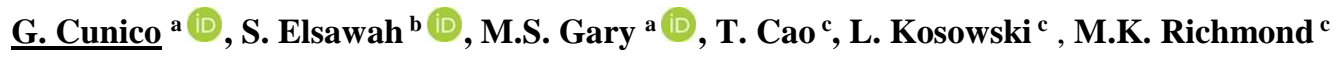 \\ ${ }^{a}$ Business School, University of New South Wales (UNSW), Sydney, New South Wales, Australia, ${ }^{b}$ Capability \\ Systems Centre, UNSW Canberra, ${ }^{c}$ Defence Science \& Technology Group, Edinburgh South Australia \\ Email: g.cunico@unsw.edu.au
}

\begin{abstract}
System dynamics (SD) encompasses a suite of conceptual and simulation methods used to study complex system behaviour over time. The structure of an SD model represents the cause-effect relationships and feedback loops in the system. SD models include stocks and flows to map system structure. Stocks, also known as accumulations, characterise the system state and change in response to flows (also known as rates). For example, the number of armoured vehicles involved in a conflict could be represented as a stock that changes in response to the attrition rate. SD causal mapping and conceptual models help teams generate ideas and facilitate shared understanding by developing a coherent representation of the problem. The causal representation of SD models provides a useful means for stakeholders to understand dynamic problems, reason about the effect of changes, and identify potential leverage points. The use of simulation methods to examine dynamic behaviour over time enables assessing alternative decisions under a range of scenarios, evaluating the veracity of different views and providing evidence linking assumptions and outcomes.

SD has been used to analyse a range of different defence decisions and topics. In this paper, we focus on combat modelling as an important research and application area in defence modelling. Given the lack of synthesis on the use of SD in combat modelling, this paper provides a preliminary exploration of the topic to identify the most salient points and set the groundwork for future research. We review and synthesise published SD applications in combat modelling to understand how SD models have been used to analyse alternative combat force designs and strategies. The review provides a starting point for researchers who want to understand the SD combat modelling literature and those who want to build SD models to examine combat dynamics.

The paper is organised around addressing four research questions: (i) why use SD for combat modelling, (ii) where does SD combat modelling fit within the broad SD defence modelling domain, (iii) what recurrent themes and operationalisation can be found in SD combat models, and (iv) what is the SD combat modelling state of the art? We conclude by identifying promising future research directions, such as: applying SD to explain the impact of new technologies involving the integration of autonomous systems to combat.
\end{abstract}

Keywords: System dynamics, defence modelling, combat modelling 


\section{INTRODUCTION}

System dynamics (SD) encompasses a suite of conceptual and simulation methods used to study complex system behaviour over time (Forrester, 1961). The structure of an SD model represents the cause-effect relationships and feedback loops in the system. SD models include stocks and flows to map system structure. Stocks characterise the system state and change in response to flows. For example, the number of casualties during combat could be represented as a stock that changes in response to the attrition rate. SD causal mapping and conceptual models help teams generate ideas and facilitate shared understanding across multiple perspectives by developing a coherent representation of the problem. The causal representation of SD models provides a useful means for stakeholders to understand dynamic problems, reason about the effect of changes, and identify potential leverage points. The use of simulation methods to represent over time changes in problem behaviour enables assessing alternative decisions under a range of scenarios, evaluating the veracity of different views and providing evidence linking assumptions and outcomes.

SD has been used to analyse a range of different defence decisions and topics (Coyle, 1996). McLucas and Elsawah (2019) present an in-depth overview of SD studies addressing different defence topics. In contrast to existing reviews (Coyle, 1996; McLucas \& Elsawah, 2019) broadly examining the role of SD in defence, this paper focuses explicitly on combat modelling as an important research and application area in defence modelling. We review and synthesise published SD applications in combat modelling to understand how SD models have been used to analyse alternative combat force designs and strategies. We find combat modelling applications scattered widely in the form of case studies, with little integration of knowledge accumulating and with limited theoretical and methodological frameworks to guide future work.

This paper focuses on the following research questions (RQs): (i) why use SD for combat modelling (ii) where does SD combat modelling fit within the broad SD defence modelling domain, (iii) what recurrent themes and operationalisation can be found in SD combat models, and (iv) what is the SD combat modelling state of the art? Given the lack of synthesis on the use of SD in combat modelling, this paper provides a preliminary exploration of the topic to identify the most salient points and set the groundwork for future research. The article is organised as follows. First, we briefly outline the method used. Then, the answers to the four RQs are reported. Finally, we reflect on the implications of these answers and draw some conclusions.

\section{METHOD}

We conducted a literature review using two scientific databases (Google Scholar and Scopus). While Scopus provides thorough coverage of peer-reviewed literature, Google Scholar also includes unpublished papers and reports. Combat modelling is not a clear thread within the SD defence applications, so the related literature is not identifiable through just a few specific keywords. To maximize the review coverage and minimize the risk of overlooking relevant information, we searched 26 keywords or phrases, combined with the expression 'system dynamics': 'defense', 'fight', 'combat', 'war', 'battle', 'warfare', 'troop', 'Lanchester', 'security', 'conflict', 'cyber-security', 'counter-insurgency, 'insurgency', 'terrorism', 'army', 'military', 'red vs blue', 'navy', 'airforce', 'manoeuvre', 'firepower', 'tactics', 'C4ISR', 'COIN', 'capability' and 'attack'. We considered only work published in English up to April 2021.

\section{RESULTS}

\subsection{Why use SD for Combat Modelling?}

To understand how SD can complement other combat modelling approaches, we also examined current combat modelling approaches employed in Defence Science \& Technology Group (DSTG). Other modelling approaches DSTG uses include attrition-based differential equation models, high-fidelity simulation models, and Bayesian Network models. We briefly summarise these approaches and identify important limitations. The limitations associated with each approach highlight opportunities for SD to contribute new insights.

Attrition-based differential equation models represent attrition using a set of ordinary differential equations based on force loss ratio (i.e., Lanchester Equation) (Pincombe et al., 2010). This approach has greatly influenced military combat modelling for many years (Lanchester, 1916). However, an underlying assumption of these equations is that a winner is determined either by total annihilation or when force numbers reach a predetermined level. While perhaps applicable to ancient and outdated forms of warfare, this underlying assumption must be addressed if these equations are to be applied to modern warfare that relies heavily on discrepancies of terrain, communications, sensors, weapon, soldier capabilities, morale and will to fight. 
High fidelity combat simulations such as COMBATXX1 (Chau et al., 2017) are entity-level, stochastic combat models. Such models represent force-on-force scenarios down to the individual soldier, system components of land combat vehicles such as firepower and sensors, tactical decision making and environmental factors. Developing these high-fidelity models are very resource-intensive and the scope is normally limited to a small set of detailed vignettes.

Bayesian Network (BN) models examine operational effectiveness through causal relationships and conditional probabilities. It is well known that feedback loops and dynamic behaviour are not very well represented by BN (Nguyen \& Cao, 2019).

Our literature review finds that SD has been used to analyse a wide range of issues under the broad combat modelling umbrella, and that SD can help offset these limitations of other modelling approaches. SD models represent complex systems through causal representations, feedback loops, and qualitative and quantitative variables. SD modelling also focuses explicitly on dynamic behaviour. None of DSTG's current combat modelling approaches has all those combined features and therefore SD can complement other techniques.

\subsection{Where does SD Combat Modelling fit within the broad SD Defence Modelling Domain?}

Our review highlights how SD defence modelling research can be organised as a concentric set of levels of analysis, with combat modelling at the centre (Fig.1). When applied to combat - the simulation of conflict between opposing forces - SD has been used to study air, land and sea conflicts (e.g. Liu et al., 2014); soldiers' performance (e.g. Artelli at al., 2009); territory patrol and control (e.g. Coyle, 1996); command and control (e.g. Gao et al., 2015); coalition responses, international relations and alliances (e.g. Langsæter, 1984); capacity deployment and supply transportation (e.g. Tang et al., 2014); and insurgency and counter-insurgency (e.g. Choucri et al., 2007). This level of analysis is embedded within the larger research area of defence capability (McLucas \& Elsawah, 2019), which focuses on the provision of fundamental capability inputs and their preparedness. Specifically, SD models on defence capability explore (Coyle, 1996; McLucas \& Elsawah, 2019): equipment maintenance, units recruiting/training and career planning (e.g., workforce management), resources supply, material upgrade, assets acquisition, project management, capability development, operation readiness, and general defence readiness and sustainability. Moving to a higher level of analysis, SD defence models have been applied to defence analysis (or budgeting), focusing on how the amount of resources available is defined and invested and spent according to specific defence strategies (Coyle, 1996). While defence capability defines the military's ability to respond to a combat threat, it depends on the broader national defence approaches (i.e., defence analysis - budgeting). In this respect, McLucas and Elsawah (2019) remind us that larger budgets do not necessarily or always translate into improved military power. Finally, we identified an additional external level called context, referring to the national-international and economic-technological developments that generate opportunities and threats. Indeed, McLucas and Elsawah (2019, p. 6) state that "nations do not exist in isolation"; their economies are influenced by the need of providing security against domestic and international risks. Thus, the national capacity to sustain itself and guarantee security strictly depends on the economy's strength and the relationships with other nations.

These levels of analysis represent different topics and perspectives in SD defence modelling applications, but do not characterise distinct research threads with rigid boundaries. In practice, many SD models of complex defence problems integrate across these different levels of analysis. Indeed, some SD applications integrate all these levels of analysis by exploring how context affects the defence analysis and strategies, which determine the defence capability and, ultimately, the specific combat dynamics. For example, in his review, Coyle (1996) reports studies focusing on the effects of US navy growth on finance in the 1980s or on the relation between perception of threat and military capability acquisition (i.e., integrating context, defence analysis and defence capability perspectives). The ability and flexibility to examine complex dynamics across different levels of analysis can be an appealing feature of SD for defence analysts, modellers and practitioners. This ability to integrate across multiple levels of analysis contrasts with some other modelling approaches. Most conflict research and management adopt some "forms of systemic closures" to simplify the problem, isolating it from the context (Gallo, 2013, p.5). For combat modelling, integrating multiple levels of analysis may be an important strength of the SD approach. In conclusion, SD combat modelling occupies a defined space within the SD defence modelling 'ecosystem' and identifies a specific analysis level. However, given SD's inclination to embrace complexity, SD combat models often span and spill over the other levels of analysis to include elements that affect combat dynamics but belong to different analytical levels.

\section{3. $\quad$ Recurring Themes of SD Combat Modelling Applications}

Our review distinguished six main thematic areas recurring in SD in combat modelling. Fig. 1 maps the six 
themes - each theme indicated by number - to the broader context of how SD applications support defence analysis. Table 1 lists the six themes and provides example citations of case studies within each theme.

The first theme involves SD applications that focus on modelling the effects of engagement and attrition. These applications simulate combat dynamics between opposing forces, with an emphasis on understanding attrition, weapons usage, reserves deployment rates and the factors influencing those rates. The main questions addressed by applications in this theme include: (a) how many forces are needed to ensure a successful operation and when reserves should be engaged, and (b) is there a main difference between modern weapons and older weapons capabilities resulting in different engagement principles? In general, combat is often modelled in SD through the Red versus Blue conceptual scheme with a hypothetical red force attacking blue defenders. This approach often uses the Lanchester laws (Lanchester, 1916), a set of differential equations describing the strengths of two opposing armies over time, as equation formulations. SD can readily be used to model the dynamics of the Lanchester equations by simulating attrition rates during combat.

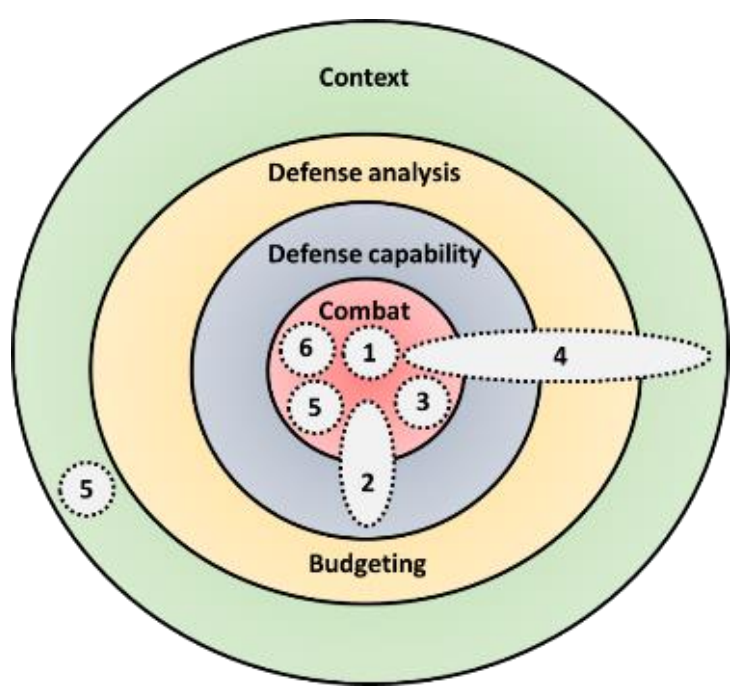

Figure 1. SD defence modelling levels of analysis and six numbered combat themes

Table 1. Examples of SD applications in combat modelling

\begin{tabular}{|c|l|l|}
\hline$\#$ & Theme & Papers \\
\hline 1 & $\begin{array}{l}\text { Modelling effects using engagement } \\
\text { and attrition }\end{array}$ & $\begin{array}{l}\text { (Chetcuti, 2020) (Coyle, 1996) (Jia et al., 2019) (Langsæter, } \\
\text { 1984) (Liu et al., 2014) (Wolstenholme, 1987) } \\
\text { (Wolstenholme \& Al-Alusi, 1987) }\end{array}$ \\
\hline 2 & Logistics and maintenance models & (Clark, 1986) (Tang, Zhou, \& Xu, 2014) \\
\hline 3 & $\begin{array}{l}\text { Modelling sensing, communication, } \\
\text { command and control }\end{array}$ & $\begin{array}{l}\text { (Chetcuti, 2020) (Coyle, 1996) (Gao et al., 2015) (Jia et al., } \\
\text { 2019) (Wolstenholme, 1987) }\end{array}$ \\
\hline 4 & Modelling insurgency operations & $\begin{array}{l}\text { (Choucri et al., 2007) (Clark, 1986) (Coyle, 1985) (Popp et } \\
\text { al., 2006) }\end{array}$ \\
\hline 5 & $\begin{array}{l}\text { Human factors affecting operations and } \\
\text { forces }\end{array}$ & $\begin{array}{l}\text { (Artelli et al., 2009) (Choucri et al., 2007) (Clark, 1986) } \\
\text { (Coyle, 1985) (Popp et al., 2006) }\end{array}$ \\
\hline 6 & Modelling movement and formation & (Langsæter, 1984) (Wolstenholme \& Al-Alusi, 1987) \\
\hline
\end{tabular}

The second theme includes logistics and maintenance models that analyse the supply chain, battlefield material maintenance, and ammunition provision lines during combat and supporting successful operations. Applications in this theme have also included models that examine insurgency activities targeting supply lines and models evaluating the risks of supply delays and disruptions (e.g. Clark, 1986).

The third theme involves modelling sensing, communication, command and control. These models represent the effects of various communication and command structures (e.g. centralised vs decentralised), information flows and the impact of delays' on decision-making. SD has been especially beneficial in capturing feedback effects in the command and control spheres to simulate how information is collected, framed and processed (Coyle, 1996).

The fourth theme comprises models of insurgency operations to understand the elements driving insurgency conflicts. This includes socio-cultural factors and their effects on force engagement. SD applications in this theme also examine the effectiveness of counter-insurgency measures.

SD applications categorised within the fifth theme examine human factors affecting operations. These models investigate how socio-cultural (e.g., public opinion) and human cognitive and/or behavioural variables (e.g., soldier morale) affect battlefield performance (e.g. Artelli et al., 2009). Models within this theme also examine how social and cultural factors influence conflict and insurgency (e.g. Popp et al., 2006). This covers a wide range of operating contexts, including counter-insurgency support, stability and reconstruction, humanitarian assistance and disaster relief. Partially, this theme may overlap with the previous one. 
The sixth theme involves modelling movement and formation. SD applications within this theme focus on capturing in an aggregated way how forces physically move in the operating environment, how this influences their actions, and how interactions with the enemy and the central command affects subsequent movement.

These six recurring themes emerged from the literature review and provide an overview of the range of combat topics for which SD models have been applied. The themes also demonstrate that SD models often integrate concepts across multiple levels of defence analysis.

\subsection{Evolution of SD Combat Modelling}

In the 1980s, SD combat modelling was typically used for system conceptualisation, for improving the understanding of combat dynamics, and for uncovering unexpected dynamic behaviours (Langsæter, 1984). The themes studied included combat engagement (Wolstenholme, 1987), alliances combat dynamics (Langsæter, 1984), insurgency analysis (Coyle, 1985) and conflict logistics and capacity maintenance (Clark, 1986). In the 1990s, limited new SD combat modelling applications emerged, and attention to the approach faded a little. By the early 2000s, SD applications to combat regained traction and much of the work focused on analysing insurgency and terrorism dynamics (Choucri et al., 2007; Popp et al., 2006). Until the mid-2010s, SD combat modelling efforts mostly concentrated on insurgency-terrorism, probably reacting to the many worldwide terrorist incidents. More recently, the focus has shifted to modelling sensing, communication, command and control (Chetcuti, 2020; Jia et al., 2019). This new generation of SD combat models includes examining how new technologies, such as unmanned vehicles (e.g., drones), affect combat dynamics and logistics. This trend relates to the emergence of new technologies and warfare approaches.

Over the years, model optimisation has gained particular attention in SD research on defence (e.g., expenditure and resource optimisation), including combat modelling (Coyle, 1996). Optimisation analysis has focussed on finding the optimal resource allocation for reducing mobilisation delays, allocating targets to land combat and air assets, and optimising synergies with allies (Coyle, 1996). Besides the usual benefits of optimisation, optimisation analyses using SD models improve the understanding of nonlinearities. For example, Wolstenholme and Al-Alusi (1987) show how optimisation can be applied to identify the nonlinear policies used by an attacker and found that the optimal policy responses were very different from those initially chosen. Also, McLucas and Elsawah (2019) review studies that use SD to optimise across the whole defence system that yields better outcomes than optimising for sub-parts of the system.

Unfortunately, our review also raised concerns over the average quality of published SD combat models. Many of the papers reviewed do not provide the necessary documentation or follow the transparency guidelines and minimum standards increasingly demanded by the broader modelling community (Martinez-Moyano, 2012; Sterman \& Rahmandad, 2012). This undermines replicability, reliability and reusability. Moreover, validation is often not discussed in-depth or only limited to behavioural replication; which alone may not give enough confidence on the model reliability and does not apply the large set of validation methods available (Barlas, 1996). Overall, these findings align with the concerns about defence model quality raised by Jnitova et al. (2017), highlighting the limited attention to use and/or report on the model validation process and outcomes. In addition, extensive use of equation formulations that include discontinuities and discrete thresholds raise concerns because such formulations do not adequately represent the nonlinear responses of the real system (e.g. Chetcuti, 2020; Liu et al., 2014). When simulated, such formulations produce spiky and sharp-edged dynamic behaviour that rarely matches actual system behaviour. More importantly, using models that include such formulations to identify high-performing policies and strategies may yield incorrect solutions because the model may misspecify important nonlinearities (Forrester, 1961).

\section{DISCUSSION AND CONCLUSION}

Within the broad operations research toolbox of modelling methods used to analyse defence problems, SD plays an important role. However, an in-depth review of SD applications for combat modelling did not exist. To close this gap, we reviewed the literature on SD combat models and three general insights emerged. First, SD defence modelling encompasses multiple levels of analysis that can be conceptualised as a series of concentric circles in which combat modelling is at the centre (Fig. 1). Second, we identified six recurring themes to classify SD combat modelling applications. Third, we observed how the focus of attention on particular combat-related topics has changed over time, with the most recent work investigating how contemporary warfare dynamics are affected by new technologies. One promising future research area applying $\mathrm{SD}$ is to explain the impact of new technologies involving the integration of autonomous systems to combat. SD could be used to represent the causal relationships and feedback interactions among the human (e.g. trust) 
and technological (e.g. level of automation) elements, and how these could affect the mission performance (Cummings \& Clare, 2015).

Our review also helped to identify the strengths of the SD method for combat modelling. The SD approach enables modellers to capture multiple levels of analysis, to represent soft variables (Clare, 2013; Hussein et al., 2019), and to optimise over the entire system rather than the parts of the system. In addition, the SD modelling approach can represent a strategic perspective of combat using relatively small models. SD demands a broader point of view than is usually found in combat and war simulations, which tend to emphasise the fine details of attrition (Coyle, 1996). In this way, SD encourages a wider view in which to analyse combat dynamics. For example, it is possible to capture in the same SD model "combat attrition, force strength, the logistics of movement, the transmission of information and the concepts of command" (Coyle, 1996, p. 142). Thus, SD fosters a more strategic perspective through the usage of small models and diagrams, compared to other defence modelling efforts, and may generate insights more easily and quickly than comparatively large models developed using alternative approaches (Wolstenholme, 1988; Coyle, 1996).

Our review also shows that SD combat modelling applications have not yet evolved into a mature line of research. For instance, we had to search for many different keywords because researchers classify SD combat applications in many different ways and often use different terminology. Even within each of the six themes we identified, researchers may not see themselves contributing to a common thread. This creates challenges for building on and extending previous work to cumulate knowledge, but also signals opportunities for future research to draw these disparate threads together into vibrant research communities. Once these communities form, debates about the weaknesses we identified in SD combat models - such as low levels of transparency, full model documentation, open-source availability of model files and data used to calibrate the models, and use of discontinuous equation formulations - can begin. Such research debates and discussions can lead to improvements in future model quality, accuracy and usefulness. Finally, the debates should further reflect on the strengths of SD for combat modelling. This would clarify the benefits of using SD versus other modelling approaches or in combination with other approaches in the defence domain and promote greater usage.

An enhanced debate on the topic could lead to the fruitful transfer of learning from SD applications in other domains to SD combat modelling. For example, while gamification of SD models in other disciplines such as environmental or supply chain modelling is common (Cunico et al., 2021), we found limited use of gamification in published SD applications of defence and combat modelling. We only found one case reporting a gamification attempt. Bakken and Gilljam (2003) developed a simulation game ('microworld') in which the player, acting as the commander, is demanded not only to develop and execute their military plans but to manage the resources necessary for the execution. The gamification of SD combat models could yield significant impacts in terms of both users (e.g., knowledge and skills acquisition) and researchers (e.g., experimental platforms). We also did not find any structured participatory modelling efforts. Many SD applications in other domains involve stakeholders and decision-makers in model development. This impacts quality, creativity, consensus, acceptance and strategy implementation and helps elicit decision-makers mental models (McLucas \& Elsawah, 2019; Vennix, 1996). Such benefits can be important in defence contexts. Finally, SD could be part of a broader combat modelling strategy integrated with other methods. For example, SD could be combined with Geographic Information System for battlefield representation. The integration of SD and other modelling paradigms, such as Discrete-Event Simulation and Agent-Based Modelling, in a hybrid modelling framework provides a promising opportunity for future research in combat modelling.

This paper provides a starting point for researchers who want to understand the SD combat modelling literature and those who want to build SD models to examine combat dynamics.

\section{ACKNOWLEDGEMENTS}

This project is funded by DSP Bilateral Collaborative Project Agreement with University of New South Wales (Agreement No: 10287) in relation to Future Land Combat Team Dynamics: Applying system dynamics to analyse new technology concepts.

\section{REFERENCES}

Artelli, M.M.J., Deckro, R.F., Zalewski, C.D.J., Leach, M.S.E., Perry, M.B., 2009. A system dynamics model for selected elements of modern conflict. Mil. Oper. Res. 14, 51-74.

Bakken, B.T., Gilljam, M., 2003. Dynamic intuition in military command and control: why it is important, and how it should be developed. Cogn. Technol. Work 5, 197-205.

Barlas, Y., 1996. Formal aspects of model validity and validation in system dynamics. Syst. Dyn. Rev. 12, $183-210$. 
Borger, J., 2010. Afghanistan: the PowerPoint solution. Guardian.

Chau, W., Gill, A. and Grieger, D. 2017. Using Combat Simulation and Sensitivity Analysis to Support Evaluation of Land Combat Vehicle. 22nd International Congress on Modelling and Simulation, Hobart, Tasmania, Australia, 3 to 8 December 2017

Chetcuti, S.C., 2020. A Framework for Developing Executable Architecture for Aerial Intelligence Surveillance and Reconnaissance Systems-of-Systems: a System Dynamics Approach. Georgia Tech

Choucri, N., Goldsmith, D., Madnick, S.E., Mistree, D., Morrison, J.B., Siegel, M., 2007. Using System Dynamics to Model and Better Understand State Stability. SSRN Electron. J.

Clare, A. (2013). Modeling Real-Time Human-Automation Collaborative Scheduling of Unmanned Vehicles. Massachusetts Institute of Technlogy. Retrieved from https://dspace.mit.edu/handle/1721.1/82469

Clark, T.D., 1986. Applications and Implementation A Management Systems Analysis of the Arms Transfer Process. Decis. Sci. 17, 517-557.

Coyle, R.G., 1996. System dynamics applied to defense analysis: A literature survey. Def. Anal. 12, 141-160.

Coyle, R.G., 1985. A system description of counter insurgency warfare. Policy Sci. 18, 55-78.

Cummings, M. L., \& Clare, A. S. (2015). Holistic modelling for human-autonomous system interaction. Theoretical Issues in Ergonomics Science, 16(3), 214-231. https://doi.org/10.1080/1463922X.2014.1003990

Cunico, G., Aivazidou, E., Mollona, E., 2021. System dynamics gamification: A proposal for shared principles. Syst. Res. Behav. Sci. sres.2805.

Forrester, J., 1961. Industrial Dynamics. Massachusetts Institute of Technology Press.

Gallo, G., 2013. Conflict Theory, Complexity and Systems Approach. Syst. Res. Behav. Sci. 30, 156-175.

Gao, D., Huang, J., Yuhu, Xu, G., Zhang, N., 2015. Research on combat SD model based on OODA loop, in: Cheng Y. Dai Y., L.S. (Ed.), Proceedings - 2015 2nd International Conference on Information Science and Control Engineering, ICISCE 2015. Institute of Electrical and Electronics Engineers, pp. 884-888.

Jia, N., Yang, Z., Yang, K., 2019. Operational Effectiveness Evaluation of the Swarming UAVs Combat System Based on a System Dynamics Model. IEEE Access 7, 25209-25224.

Jnitova, V., Elsawah, S., Ryan, M., 2017. Review of simulation models in military workforce planning and management context. J. Def. Model. Simul. 14, 447-463.

Lanchester, F.W., 1916. Aircraft in Warfare: The Dawn of the Fourth Arm. Constable Limited, London, UK.

Langsæter, T., 1984. The Land Operations Model - An Application of System Dynamics for Modelling Land Combat on the Division Level, in: Systems Analysis and Modeling in Defense. Springer US, Boston, MA, pp. 123-138.

Liu, P.-L., Sun, H.-K., You, Y.-T., 2014. A system dynamics model for modern AirLand battle. Asia-Pacific J. Oper. Res. 31, 1450031.

Martín-Martín, A., Orduna-Malea, E., Thelwall, M., Delgado López-Cózar, E., 2018. Google Scholar, Web of Science, and Scopus: A systematic comparison of citations in 252 subject categories. J. Informetr. 12, 1160-1177.

Martinez-Moyano, I.J., 2012. Documentation for model transparency. Syst. Dyn. Rev. 28, 199-208.

McLucas, A.C., Elsawah, S., 2019. System Dynamics Modeling to Inform Defense Strategic Decision-Making, in: Encyclopedia of Complexity and Systems Science. Springer Berlin Heidelberg, pp. 1-33.

Nguyen, M.-T. and Cao, T. 2019, 'A multi-method approach to evaluate land combat vehicle system', International Journal of Applied Decision Sciences 12(4), 337-360.

Pincombe, A., Pincombe, B. and Pearce, C.E.M. 2010, A simple battle model with explanatory power. ANZIAM J. 51 (EMAC2009) pp.C497-C511.

Popp, R., Kaisler, S.H., Allen, D., Cioffi-Revilla, C., Carley, K.M., Azam, M., Russell, A., Choucri, N., Kugler, J., 2006. Assessing Nation-State Instability and Failure, in: 2006 IEEE Aerospace Conference, pp. 1-18.

Sterman, J., Rahmandad, H., 2012. Reporting guidelines for simulation-based research in social sciences. Syst. Dyn. Rev. 28, 396-411.

Tang, J., Zhou, Q., Xu, P., 2014. US army aviation's battlefield POL assurance model simulation based on system dynamics. Comput. Model. New Technoligies 18, 28-33.

Vennix, J.A.M., 1996. Group model building. Wiley, Chichester.

Wolstenholme, E.F., 1987. A systems approach to combat modelling. Trans. Inst. Meas. Control 9, 45-51.

Wolstenholme, E.F., Al-Alusi, A. -S, 1987. System dynamics and heuristic optimisation in defence analysis. Syst. Dyn. Rev. 3, 102-115. 